\title{
A Case of Extensive Grover's Disease in a Patient with a History of Multiple Non-Melanoma Skin Cancers
}

\author{
Mareike Kotzerke Fouad Mitri Alexander Enk Ferdinand Toberer \\ Holger Haenssle
}

Department of Dermatology, University Hospital Heidelberg, Heidelberg, Germany

\section{Keywords}

Grover disease · Transient acantholytic dermatosis · Squamous cell carcinoma

\begin{abstract}
We report on a 69-year-old man who presented with itching and erythematous papules on his torso and extremities, which were resistant to topical therapy with antibiotics and steroids. Physical examination revealed multiple erythematous papules on his back, neckline, and lower extremities. The lesions had appeared 4 years earlier and usually worsened with heat or extensive sweating. Histopathology of previous skin biopsies had shown multiple cutaneous squamous cell carcinomas or was non-conclusive. Thus, a re-biopsy was performed, revealing acanthosis and focal acantholytic dyskeratosis. These clinical and anamnestic findings lead to the diagnosis of extensive Grover's disease (GD). Oral therapy with isotretinoin 30-mg QD led to the regression of the skin lesions. Topical adapalene, as well as topical corticosteroids, were later prescribed for maintenance therapy.
\end{abstract}

\section{Case Report}

A 69-year-old Caucasian man presented to our outpatient department with a pruritic papular rash on his torso and extremities that was resistant to local therapy with antibiotics and steroids. The skin lesions initially appeared 4 years ago and tended to worsen under 
exposure to heat and increased sweating. In the past, multiple biopsies were submitted to histopathological workup, leading to the diagnosis of multiple cutaneous squamous cell carcinomas followed by further excisions. Besides, his skin lesions the medical history of the patient was unremarkable. Moreover, his family history was negative. B symptoms were not present, the initial blood workup showed no abnormalities, and the skin cancer screening was inconspicuous. Physical examination revealed erythematous, focally eroded papules on his back, neckline, and lower extremities, as well a fine-lamellar scaling on the extremities and erythematous plaques on his left hand (Fig. 1a, c). On dermoscopy, yellow crypts with white halos in a radiating pattern were observed (Fig. 1e). Due to the suspicious medical history and the clinical presentation, a re-biopsy was performed. Histopathologically, acanthosis, focal acantholytic dyskeratosis, and parakeratosis as well as a superficial, perivascular, lymphohistiocytic infiltrate was seen (Fig. 2). Based on the negative family history for hereditary diseases, the chronic course of disease, worsening under exposure to heat and sweating, as well as the clinical and histologic characteristics, extensive Grover's disease (GD) was diagnosed. We treated our patient with oral isotretinoin $30 \mathrm{mg}$ QD for 2 months and $40 \mathrm{mg}$ QD for 4 months. The skin lesions improved significantly under this treatment (Fig. 1b, d); however, due to extensive myalgia and back pain, the medication had to be discontinued. Since then, the patient received local adapalene and if needed local corticosteroids for maintenance therapy.

\section{Discussion and Conclusion}

GD was first described in 1970 as transient acantholytic dermatosis [1] typically occurring in white men around the sixth decade of life [2]. It clinically presents with benign, polymorphic, papulovesicular eruptions on the trunk that can form crusts and keratotic erosions [3]. In case of involvement of at least 1 additional body area, GD is classified as extensive or atypical if it involves a non-truncal area [4] as the case in our patient. The predominant symptom in $75 \%$ of GD patients is pruritus, whereas $25 \%$ are asymptomatic [2], the latter frequently being reported in extensive cases with associated malignancy [4]. The etiology of GD is unknown; however, several factors promoting the onset or extent of the disease have been reported. Heat and/or excessive sweating and cold and dry air as well as exposure to UV light are commonly associated with worsening of symptoms in cases of typical GD [5], whereas factors like underlying malignancy, chemotherapy, or organ transplantation are more common in extensive and atypical cases [4]. The most commonly associated malignancies in extensive cases include hematologic cancers (65\%) and solid tumors (32\%) with an emphasis on metastatic melanoma [4]. Further reported associations with GD include renal failure, the initiation of hemodialysis [6], other dermatologic conditions such as allergic contact dermatitis, atopic dermatitis [7] or scabies [8], as well as several medications, for example, BRAF inhibitors [9] or immune checkpoint inhibitors [10]. Interestingly, there is usually a latency period of several weeks from exposure to disease development. Since all the abovementioned factors lead to a nonspecific irritation or immune-altered status of the skin, Aldana and Khachemoune [5] hypothesized that GD results from the change in the integrity of the skin rather than from direct epidermal destruction, for example, through significant xerosis as the case in renal failure [6].

Fig. 1. Clinical pictures and dermoscopy. a, c Physical examination reveals erythematous eroded papules on the back and lower extremities of a patient with GD. b, d Progress 6 months after therapy with isotretinoin. e A central, stellar-like, yellowish keratotic plug with a white radiating rim along with linear and/or hairpin blood vessels. GD, Grover's disease.

\section{Karger'}

(For figure see next page.) 
Case Reports

in Dermatology
Case Rep Dermatol 2021;13:553-557

DOI: 10.1159/000519168

C 2021 The Author(s). Published by S. Karger AG, Basel www.karger.com/cde

Kotzerke et al.: A Case of Extensive Grover's Disease

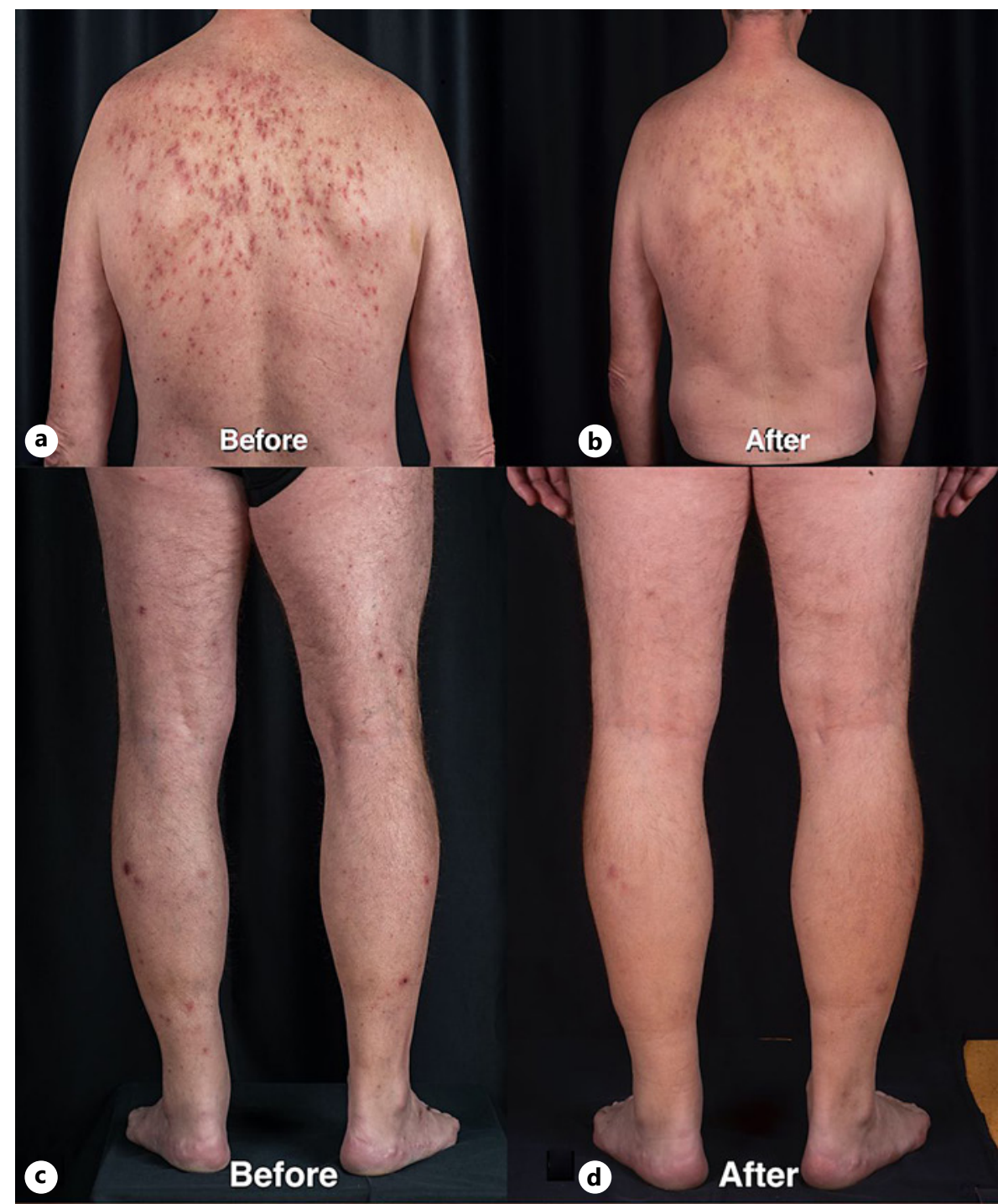

e

\section{Karger'}



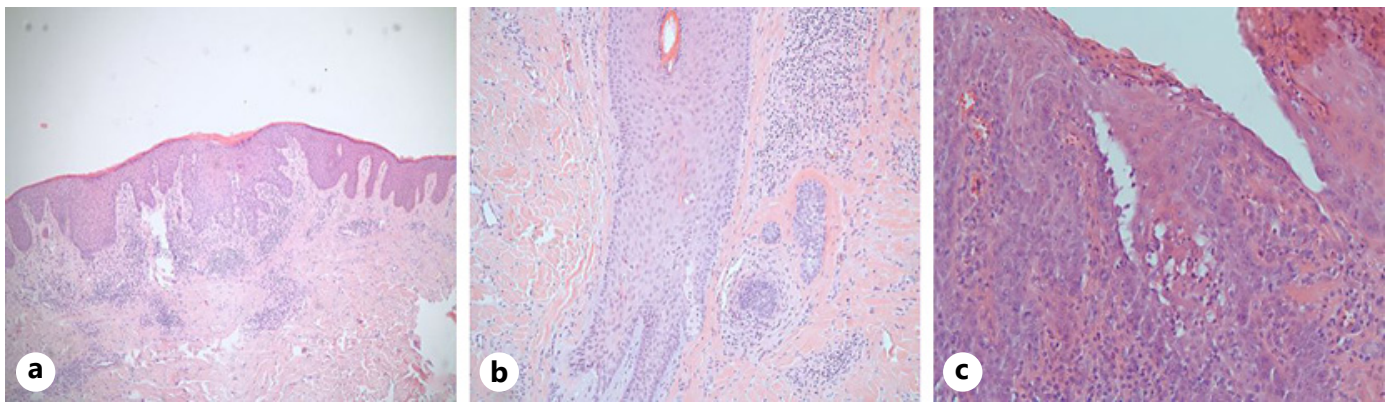

Fig. 2. Histopathology. a Section showing an overview of the skin (hematoxylin and eosin stain. $\times 25$ ). Section revealing dyskeratotic keratinocytes (hematoxylin and eosin stain, magnification $\times 100$ ) (b), close-up view showing acantholysis (hematoxylin and eosin stain, magnification $\times 200$ ) (c).

Diagnosis is often made at a late stage [3] and clinical features, as well as histology, are often needed. Nevertheless, distinct dermoscopic features of early and late-stage GD can be helpful in uncertain cases. Dermoscopically, GD lesions usually evolve from an unspecific initial appearance showing a pinkish-white background with polymorphous vessels to latestage lesions characterized by a pink-yellow background, a central, stellar-like, yellowish keratotic plug with a white radiating rim along with linear and/or hairpin blood vessels [11, 12] as seen in our patient (Fig. 1e).

From a histopathological perspective, there are 4 classical patterns: Darier-like, Hailey-Hailey-like [1], pemphigus-vulgaris like, and spongiotic-like [13], which can occur separately or simultaneously. All variants share the common distinguishing features of acantholysis and dyskeratosis [5] (Fig. 2). Recently, a dysmaturative pattern in GD, characterized by epithelial buds with atypical keratinocytes, was described $[14,15]$. This might explain why some small biopsies of GD could be mistaken for malignant epithelial lesions such as actinic keratosis or even Bowen's disease.

The course of the disease can vary from a spontaneous resolution without treatment (in up to $42 \%$ of cases) [2] to a recurrent or chronic course in which skin lesions and pruritus last for several years with little response to therapy [5].

First-line therapy of GD includes topical steroids, vitamin D analogs, and adjuvant oral antihistamines for the symptomatic relief of pruritus. In severe or persistent cases systemic corticosteroids, retinoids, and phototherapy can be used. Additionally, it seems essential to strictly avoid exposure to the abovementioned promoting factors. Spontaneous regression of signs and symptoms may also occur by treatment of underlying diseases [2, 5]. The latter not only supports the pathophysiological hypothesis of GD as a secondary disease requiring predisposing conditions to occur [5] but also highlights an important treatment target: the restoration and stabilization of skin integrity through elimination of the primary source of irritation. In conclusion, physicians involved in the treatment of GD should be aware of its fluctuating course and distinct dermoscopic features which facilitate the diagnosis and should, whenever possible in an interdisciplinary approach, explore the presence of accompanying and easily treatable concomitant disorders.

\section{Statement of Ethics}

The patient in this manuscript has given written informed consent to the publication of his case details. The paper is exempt from Ethical Committee approval since it is a retrospective report on a single case.

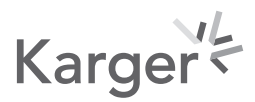




\section{Conflict of Interest Statement}

The authors have no conflicts of interest to declare.

\section{Funding Sources}

There are no funding sources which contributed relevantly to the writing process of this manuscript.

\section{Author Contribution}

Mareike Kotzerke and Fouad Mitri both contributed equally as first authors to the manuscript by acquiring and analyzing patient data, drafting the manuscript, and submitting the final version. Alexander Enk, Ferdinand Toberer, and Holger Haenssle made substantial contributions to the interpretation of data and revising process.

\section{Data Availability Statement}

All data generated or analyzed during this study are included in this article. Further inquiries can be directed to the corresponding author.

\section{References}

1 Grover RW. Transient acantholytic dermatosis. Arch Dermatol. 1970;101(4):426-34.

2 Bellinato F, Maurelli M, Gisondi P, Girolomoni G. Clinical features and treatments of transient acantholytic dermatosis (Grover's disease): a systematic review. J Dtsch Dermatol Ges. 2020;18(8):826-33.

3 Weaver J, Bergfeld WF. Grover disease (transient acantholytic dermatosis). Arch Pathol Lab Med. 2009; 133(9):1490-4.

4 Gantz M, Butler D, Goldberg M, Ryu J, McCalmont T, Shinkai K. Atypical features and systemic associations in extensive cases of Grover disease: a systematic review. J Am Acad Dermatol. 2017;77(5):952-e1.

5 Aldana PC, Khachemoune A. Grover disease: review of subtypes with a focus on management options. Int J Dermatol. 2020;59(5):543-50.

6 Casanova JM, Pujol RM, Taberner R, Egido R, Fernández E, Alomar A. Grover's disease in patients with chronic renal failure receiving hemodialysis: clinicopathologic review of 4 cases. J Am Acad Dermatol. 1999;41(6): 1029-33.

7 Grover RW, Rosenbaum R. The association of transient acantholytic dermatosis with other skin diseases. J Am Acad Dermatol. 1984;11(2 Pt 1):253-6.

8 Kaddu S, Müllegger RR, Kerl H. Grover's disease associated with Sarcoptes scabiei. Dermatology. 2001;202(3): 252-4.

9 Carlos G, Anforth R, Clements A, Menzies AM, Carlino MS, Chou S, et al. Cutaneous toxic effects of BRAF inhibitors alone and in combination with MEK inhibitors for metastatic melanoma. JAMA Dermatol. 2015;151(10):1103-9.

10 Munoz J, Guillot B, Girard C, Dereure O, Du-Thanh A. First report of ipilimumab-induced Grover disease. Br J Dermatol. 2014;171(5):1236-7.

11 de Abreu L, Guimarães Cordeiro NG, Buçard AM, Quintella DC, Argenziano G. Dermoscopy of Grover disease. J Am Acad Dermatol. 2017;76(2s1):S60-s3.

12 Fink C, Toberer F, Haenssle HA. Dermoscopic characteristics of transient acantholytic dermatosis (Grover's disease). Eur J Dermatol. 2017;27(6):648-50.

13 Chalet M, Grover R, Ackerman AB. Transient acantholytic dermatosis: a reevaluation. Arch Dermatol. 1977; 113(4):431-5.

14 Fernandez-Figueras MT, Puig L, Cannata P, Cuatrecases M, Quer A, Ferrandiz C, et al. Grover disease: a reappraisal of histopathological diagnostic criteria in 120 cases. Am J Dermatopathol. 2010;32(6):541-9.

15 Aljarbou OZ, Asgari M, Al-Saidi N, Silloca-Cabana EO, Alathamneh M, Sangueza OP. Grover disease with epidermal dysmaturation pattern: a common histopathologic finding. Am J Dermatopathol. 2018;40(9):642-6. 